by the author himself), this chapter is particularly worthwhile reading. We might also mention the extensive bibliography.

HANS FreudenthaL

Qualitative theory of differential equations. Parts I and II. By V. V. Nemytskii and V. V. Stepanov. Trans. from the 2d Russian edition. Princeton University Press, $1960.8+523$ pp. $\$ 12.50$.

\title{
Review of Part I
}

This is a translation of the second (1949) Russian edition. There are four chapters, a bibliography and index to part one.

Chapter one is on existence and continuity theorems. It contains existence and uniqueness theorems for real systems and includes dependence on initial conditions, but not on parameters. The last section is on fields of lineal elements.

Chapter two is on systems of two differential equations and contains 110 pages. Aside from a detailed treatment of singular points in the plane, results of Poincaré and Bendixon and many extensions are given. Trajectories on a torus are treated. The Lienard plane is considered.

Chapter three is on systems of $n$ equations. It contains a treatment of linear systems, including the case of constant and periodic coefficients. Asymptotic behavior of linear and non-linear systems is treated. The main tool is the "variation of constants" formula in its matrix form.

Chapter four is on neighborhoods of singular points and of periodic solutions. The singular point in the analytic case is treated at length. Lyapunov stability and the method of surfaces of section are treated.

The appendix is an excerpt from the Bulletin of Moscow University; No. 8 (1952), Mathematics, by Nemytskii and is a survey of contributions by Russians.

(The results of O. Dunkel published in Proc. Amer. Acad. Arts Sci. vol. 38 (1912-1913) which are being rediscovered in various countries every few years appear as a relatively recent Russian result here. Since the reviewer also "discovered" the Dunkel results some years ago, he feels free to point out fellow offenders.)

NoRMAN LEVINSON

\section{Review OF PART 2}

An autonomous system of ordinary differential equations

$$
\frac{d x_{i}}{d t}=f_{i}\left(x_{1}, \cdots, x_{n}\right) \quad(i=1, \cdots, n)
$$


determines, under reasonable hypotheses, a one-parameter group of transformations acting on the common domain of the functions $f_{i}$. This fact suggests the definition of a dynamical system as a oneparameter group of transformations acting on a metric space, called the phase space. Thus, subject to certain axioms, a dynamical system assigns to each real number a homeomorphism of the phase space onto itself. The topological aspects of dynamical systems are studied in topological dynamics. The system $\left({ }^{*}\right)$ may be such that $n$-dimensional volume, or some other integral, is invariant under all transformations determined by $\left({ }^{*}\right)$. This fact suggests the postulation or construction of an invariant measure in the phase space of a dynamical system. The measure theoretic aspects of dynamical systems are studied in ergodic theory.

Chapter 5, entitled General theory of dynamical systems and consisting of 118 pages in translation, is devoted to topological dynamics. Chapter 6, entitled Systems with an integral invariant and consisting of 95 pages in translation, is on ergodic theory. Section headings in Chapter 5 are: metric spaces (appearing in the first Russian edition, but not the second), general properties and local structure of dynamical systems, $\omega$ - and $\alpha$-limit points, stability according to Poisson, regional recurrence and central motions, minimal center of attraction, minimal sets and recurrent motions, almost periodic motions, asymptotic trajectories, completely unstable dynamical systems, dynamical systems stable according to Lyapunov. Section headings in Chapter 6 are: definition of an integral invariant, Carathéodory measure, recurrence theorems, theorems of E. Hopf, G. D. Birkhoff's ergodic theorem, supplements to the ergodic theorem, statistical ergodic theorems, generalizations of the ergodic theorem, invariant measures of an arbitrary dynamical system. The bibliography to Chapter 5 contains 38 items, and the bibliography to Chapter 6 contains 29 items. There are 7 line drawings ("phase portraits") in Chapters 5 and 6, and a one-page index to Chapters 5 and 6. A numbering system for subsections, not in the second Russian edition, makes for easy reference and good appearance. Typography is excellent.

Comparison with the second Russian edition shows that the English edition is an accurate, but not always literal, translation, and that it is quite complete except for a very involved and rather incomprehensible example due to A. G. MaY̌er which appears at the end of $\$ 5$ of Chapter 5 in the second Russian edition. The example offers an autonomous system of three ordinary differential equations defined on a solid torus in 3-space whose right-hand sides are Lipschitzian and whose ordinal number of central motions exceeds an arbitrary preassigned transfinite number of the second class. 
Chapters 5 and 6 provide attractive uncomplicated introductions to topological dynamics and ergodic theory. These chapters can be read quite independently of the preceding chapters. Exposition proceeds leisurely with copious detail generously supplied. All requisite topology, which is simply that of metric spaces, is developed in $\$ 1$ of Chapter 5 starting with the definition of metric space. A similar brief development of Carathéodory measure occurs in $\$ 2$ of Chapter 6. The corresponding abstract Lebesgue integral is defined but its properties are not systematically discussed, although a needed form of Fubini's theorem is proved. Consequently, a prior acquaintance with Lebesgue integration, at least on the line, is a practical necessity for most of Chapter 6. Generality is not pursued so that motivation is usually at hand. Measure theoretic considerations are mostly tied in with topological considerations. Examples are almost all drawn from differential equations. A notable exception is Markov's example in a function space of a minimal set which is not strictly ergodic. The book is a textbook for a graduate course, but much of Chapter 5 and some of Chapter 6 could probably be understood by a talented advanced undergraduate mathematics major and would provide good material for supervised reading by such a student.

A count of the bibliography shows that 3 references are dated before 1920,7 in the decade $1920-1929,46$ in the decade $1930-1939,10$ in the decade 1940-1949, and only one after 1949. Chapters 5 and 6 are thus an exposition of results from the "classical period" of topological dynamics and ergodic theory, describing pioneering work of Poincaré and G. D. Birkhoff and contributions by von Neumann, Bebutov, Hilmy, Eberhard Hopf, Khintchine, Kryloff and Bogoliuboff, Markov, Nemytskii, Stepanov, and others. Even in 213 pages a selection must be made. For example, the symbolic dynamics of Morse and Hedlund (from the period 1920-1940, say) is not represented. Actually, discrete flows (that is, single homeomorphisms) are not considered at all, attention being confined to continuous flows. Geodesic and horocycle flows are not discussed.

Present day research in topological dynamics and ergodic theory is much concerned with structures like topological groups of homeomorphisms on topological spaces, measurable transformations on abstract measure spaces, and linear operators on Banach spaces. These things do not appear in the book because of the nature of the book. However, for anyone who wants to begin the study of topological dynamics and ergodic theory the present volume is a good starting point.

Suggested metatheorem: Mathematics is mixing. As a metacorollary, differential equations theory, topology and measure theory 
should become more and more intertwined. This book will help to accelerate the mixing process.

\section{W. H. Gottschalk}

Probability theory. By Michel Loève. 2d ed. Princeton, D. Van Nostrand Co., Inc. $16+685$ pp. $\$ 14.75$.

The second edition differs from the first principally in the addition of about 150 pages on continuous parameter stochastic processes. According to the first edition preface, calculus was a prerequisite. The second edition prerequisite is "honest" calculus. A student whose calculus course has been honest enough to prepare him for this difficult book is indeed fortunate. Even with his new section, Loève keeps to his original optimistic estimate that the material can be covered in about three to four semesters. However there is one concession: he simplifies at least his preface by warning the unlearned reader to be armed with "patience, pen, and calculus" instead of the first edition's "patience, pebble, and reed."

Loève's first edition marked the end of a golden research era in probability theory, in which the subject had been advancing too fast to be treated by anything but research articles, specialized or research books, and elementary texts. He was the first to attempt a text covering the most significant aspects of the subject in a rigorous manner. Even the over 600 pages of the second edition have of course necessarily omitted many things of interest, although the exceedingly compact style sometimes goes beyond letting the reader see beauty bare, in fact forcing him to guess at the beauty from the skeleton. But he has shown it possible to write a reasonably complete text as a central core, from which specialized works can radiate. His material includes far more than recent advanced texts by Fisz and Richter, written in a more leisurely style. Even though probability theory has reached the textbook stage, however, it seems premature to include as little history as Loève does. A bibliography is a poor substitute for frequent remarks on the background of the subject, including at least some dates if name-dropping is considered too hazardous.

Loève's new section has two chapters, both under the general heading "Elements of random analysis": XI Foundations; martingales and decomposability; XII Markov processes. "Foundations" contains a discussion of separability of processes, sample function continuity, and related topics. "Martingales" discusses martingales. "Decomposability" discusses what are called by various authors "integrals with independent elements," "additive processes," "differential processes," "processes with independent increments" and now 